\title{
Estimation of Vehicle Side-Slip Angle Using an Artificial Neural Network
}

\author{
Daniel Chindamo ${ }^{1}$ and Marco Gadola ${ }^{1}$ \\ ${ }^{1}$ Department of Industrial and Mechanical Engineering, Automotive Group, University of Brescia, I-25123 Brescia, Italy.
}

\begin{abstract}
In this work, a reliable and effective method to predict the vehicle side-slip angle is given by means of an artificial neural network. It is well known that artificial neural networks are a very powerful modelling tool. They are largely used in many engineering fields to model complex and strongly non-linear systems. For this application, the network has to be as simple as possible in order to work in real-time within built-in applications such as active safety systems. The network has been trained with the data coming from a custom manoeuvre designed in order to keep the method simple and light from the computational point of view. Therefore, a 5-10-1 (input-hidden-output layer) network layout has been used. These aspects allow the network to give a proper estimation despite its simplicity. The proposed methodology has been tested by means of the CarSim ${ }^{\circledR}$ simulation package, which is considered one of the reference tools in the field of vehicle dynamics simulation. To prove the effectiveness of the method, tests have been carried out under different adherence conditions.
\end{abstract}

\section{Introduction}

It is well known that vehicle side-slip angle is the key to understand vehicle lateral dynamics. It can be used to investigate the cornering behaviour in terms of understeer/oversteer and stability, which are related to active safety, driveability and performance, either for everyday road cars and high-performance vehicles [1, 2]. Moreover, vehicle side-slip angle is important to feed and tune active safety systems such as electronic stability program (ESP, see Table 1 for a comprehensive nomenclature), dynamic yaw control (DYC) and antilock braking system (ABS) as well as other active features like electronically controlled differentials.

Devices required to obtain a direct measure of the vehicle side-slip angle are expensive and generally difficult to fit on a car. Basically, such devices are optical transducers requiring extremely precise alignment in order to give a reliable measurement. They are also very susceptible to road surface type and weather conditions.

With regard to motorsport, racing rules often make on-board installation forbidden.

Therefore, the side-slip angle has to be estimated on the basis of conventional measurements carried out on the vehicle, i.e. lateral/longitudinal acceleration, yaw rate, steer angle and angular speed for each wheel, allowing to estimate the vehicle speed. Several side-slip angle estimation strategies have been proposed in literature. They are mainly based on Extended Kalman Filters (EKF) [3-5]. Since EKF typically requires a reference vehicle model, it is able to provide a good estimation only if model parameters are accurately known. Under this point of view, tyre characteristics are particularly critical depending on various factors like road surface conditions, temperature, tyre wear, etc.

Unfortunately, many of the parameters and data required to build the reference vehicle model are often unknown or difficult to measure, especially those referred to a key component such as the tyres. In fact, obtaining tyre data (i.e. Pacejka coefficients) from tyre manufacturers is often a real challenge.

Hence, in this paper the need of a deep vehicle knowledge has been avoided by using an artificial neural network (ANN) instead. In fact, using ANN the vehicle can be considered as a black box system and only a conventional set of sensors is needed to train/feed the network.

Artificial neural networks are considered effective tools for system modelling. Neural networks also offer decisive advantages such as adaptive learning, fault tolerance and generalization. Moreover, in recent years, the development of high speed computers encouraged the application of artificial neural networks which have been progressed very quickly.

Thus, among the others, ANNs have been largely applied also in technical disciplines like automotive engineering.

This method has been used, among others, in [6] to build a hybrid vehicle power management strategy, in [7] to model Diesel engine gas emissions, in [8] to estimate the position of an autonomous vehicle, in [9] for a longitudinal wheel slip controller, in [10] to monitor a Racecar driver vital parameters.

Therefore, literature shows that artificial neural networks are suitable to model complex systems using 
their ability to identify relationships from input-output data [11].

Some attempts of applying a neural network technique to vehicle side-slip angle estimation can also be found in literature. In $[12,13]$, the side-slip angle is estimated as a function of lateral acceleration and yaw rate. The results seem to be promising, but the effects of vehicle speed variations hence tyre-road friction are not addressed. The neural network suggested in [14] is instead based on a non-conventional set of sensors (measurements provided by four two-axis accelerometers placed on each corner of the car body are required).

Moreover, promising results have been obtained also in [15] and [16]. However, a previous estimation of the side-slip angle as input to the network (feedback) along with a large number of inputs (i.e. 9 inputs leading to many more calculations), are needed to let the ANN work properly. Hence, more calculation and CPU usage are required to deal with the network complexity.

Therefore, the aim of the present work is to further investigate the application of an ANN in order to develop a lighter solution able to give a reliable and effective realtime estimation of the vehicle side-slip angle.

ANNs give better results as much as the sets of data used to train the network contain the whole possible scenario the system may have to deal with [17], thus a specific training procedure for this application has been also proposed to keep the network layout as simple as possible hence light from the computational point of view. This allows to use a network with a very low number of neurons (5-10-1 input-hidden-output layer), reducing the number of calculations needed. This is crucial for realtime operation.

Indeed, a validation of the proposed methodology has been provided. It is carried out by means of the CarSim ${ }^{\circledR}$ package which is considered one of the reference tools in professional vehicle dynamics simulation. In order to simulate data coming from real-world sensors, CarSim ${ }^{\circledR}$ outputs have been added with uncertain random noise variance as successfully used in [18].

As reported in section 4, the proposed methodology leads to good results while keeping the network simple. The computational time is very low indeed therefore suitable for real-time applications.

It is worth to stress that this method takes into account the vehicle speed variation and is conceived to be fed using a simple and cheap set of sensors usually already onboard of the most recent passenger cars.

\section{Methods}

The approach used in this work is the same adopted by Parlak et al. in [19]. It uses the Levenberg-Marquardt back-propagation algorithm along with the mean squared error (MSE) performance index [20]. This BP optimization algorithm updates weights and biases of the network as presented in [21, 22]. See Figure 1 for the high-level schematics.

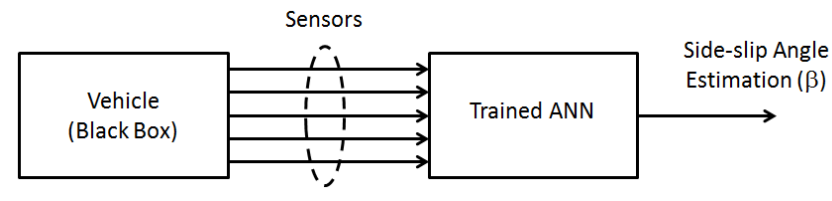

Figure 1. High-level schematics.

Then, the ANN performance has been determined by the MSE which is:

$$
M S E=\frac{1}{N} \sum_{i=1}^{N}\left(y_{i}-y_{k}\right)^{2},
$$

where $y_{i}$ and $y_{k}$ are respectively the predicted value and the target value of the $\mathrm{i}$-th pattern and $\mathrm{N}$ is the number of pattern.

The dataset required to train and test the ANN has to be composed by input data patterns together with corresponding targets. Moreover, it has to be divided in two different sets, in this case $90 \%$ of the dataset has been used to train the network while the remaining $10 \%$ has been used to test the capability of the network. This is a common procedure as reported in [23].

Required mathematical background along with testing and training procedures can be found in [24].

For this application inputs (which determine the ANN's input layer neuron number) are: vehicle speed $(v x)$, steering angle $(\delta)$, lateral acceleration (ay), longitudinal acceleration $(a x)$ and yaw rate $(\psi)$. The output is the vehicle side-slip angle $(\beta)$, hence the ANN has only one neuron in its output layer.

As stated above, this estimator is supposed to be reliable and light from the computational point of view, thus it can be used in real-time built-in applications such as vehicle active safety systems (i.e. ESP, DYC, ABS, etc.). Therefore, the neuron number of the hidden layer, which leads to satisfy both accuracy and simplicity purposes, is 10. It has been chosen using an iterative "trial and error" approach.

The resulting 5-10-1 (input-hidden-output layer) network layout has been built using the "Neural Networks Toolbox" of MATLAB ${ }^{\circledR}$. Note that, for hidden and output layer the log-sigmoid transfer function has been used.

Finally, the ANN's structure is reported in Figure 2.

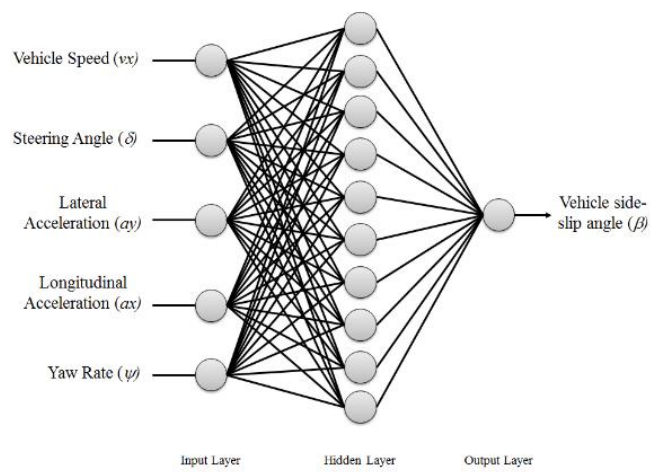

Figure 2. Layout of the adopted ANN. 


\subsection{Training data set and procedure}

The most challenging part of this work was the identification of an appropriate manoeuvre to be used for the ANN training.

As stated above, the training data set must represent as faithfully as possible the system to be simulated and its dynamics in all conditions. When this criterion is met, the neuron number of the hidden layer can be reduced (within certain limits), giving a simple and light ANN.

It is worth stressing that, for this application both the tyre linear and non-linear behaviours must be taken into account, along with the vehicle behaviour as a function of its speed. Therefore, a custom manoeuvre that explores the whole tyre range of usage has been designed.

In order to take into account the whole range of tyre slip angles, a sinusoidal steer manoeuvre has been adopted.

Hence, the proposed training manoeuvre is a $45^{\circ}$ @ $0.25 \mathrm{~Hz}$ sinusoidal steer carried out while the vehicle accelerates following a $0.25 \mathrm{~km} / \mathrm{h} / \mathrm{s}$ ramp from $5 \mathrm{~km} / \mathrm{h}$ to $100 \mathrm{~km} / \mathrm{h}$, i.e. until tyre saturation is reached.

In order to deal with all the possible meteorological conditions, such manoeuvre has been carried out with different tyre-surface friction coefficients $(\mu)$. So that, training dataset is composed by the same manoeuvre repeated for each $\mu$ (from 0.4 simulating sleet/snow conditions to 1 simulating dry surface with step increment of 0.1 ).

Indeed, the data obtained this way allows to meet the above-mentioned criteria. Moreover, in a real-world context, this manoeuvre can be easily performed by a well-trained test driver on any proving ground.

Figure 3 represents the vehicle speed and steering wheel data histories (see Figure $3 \mathrm{a}$ and $3 \mathrm{~b}$ ) of the training manoeuvre. Also the resulting vehicle side-slip angle for $\mu=1$ has been reported for reference (see Figure 3c).

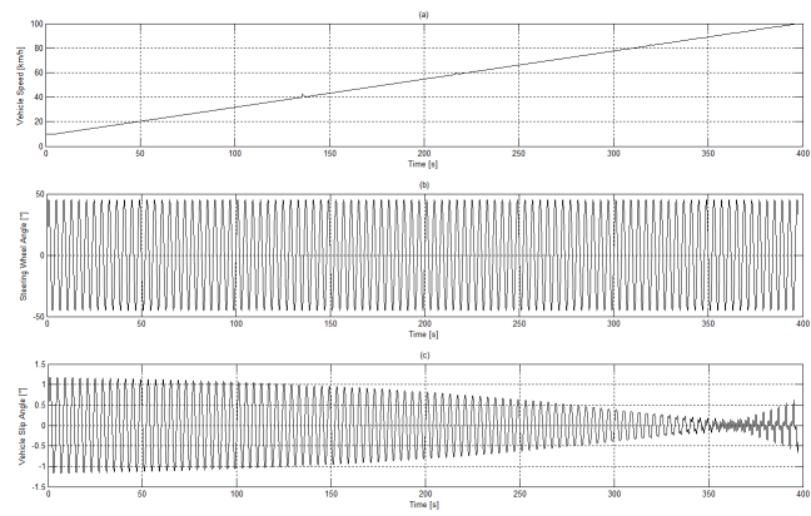

Figure 3. Adopted training manoeuvre. (a) vehicle speed, (b) steering wheel, (c) vehicle side-slip angle for $\mu=1$.

The Levenberg-Marquardt algorithm, fed with the training data set, has been able to train the network after 899 iterations, giving a final performance index (MSE) of $9.24 \mathrm{e}-6$ with a gradient of $6.82 \mathrm{e}-5$.

Therefore, the network can be considered ready to be tested with other generic data sets, as reported in the next section.

\section{Results and discussion}

As stated before, the proposed ANN has been trained and tested with a professional vehicle dynamics simulation software called $\mathrm{CarSim}^{\circledR}$ which represents a good reference in the vehicle simulation field. Many simulations were performed and compared to the results given by the network.

A GT class vehicle has been used for this purpose. Thus, a series of double lane change (DLC) manoeuvres have been simulated to check if the trained ANN was able to reproduce the vehicle lateral transient behaviour at different speeds. Also, a series of step steer inputs (SS) at different speeds have been simulated to further investigate the ANN capability and to make sure that vehicle steady state behaviour can be reproduced as well. Both DLCs and SSs have been carried out at $30 \mathrm{~km} / \mathrm{h}, 60$ $\mathrm{km} / \mathrm{h}$ and $90 \mathrm{~km} / \mathrm{h}$. All the tests have been repeated using $\mu=1$ simulating dry conditions, $\mu=0.7$ simulating heavy rain conditions and $\mu=0.4$ simulating sleet/snow conditions. It is worth stressing that, apart for the training procedure, the vehicle side-slip angle is not used as input value but just as reference. Hence, there is not feedback lines and the vehicle side-slip angle does not appear among the inputs in any form.

Test results for $\mu=1$ are reported below in figures from 4 to 9 and they show that the trained ANN gives a very good estimation especially regarding manoeuvres performed at speed below $90 \mathrm{~km} / \mathrm{h}$ where the mean error has been found to be less than $2 \%$, especially in figures 4 and 7 where the difference between the estimated and actual value of the side-slip angle is hardly visible. However, figures 6 and 9 show a good estimation for manoeuvres performed at $90 \mathrm{~km} / \mathrm{h}$ as well.

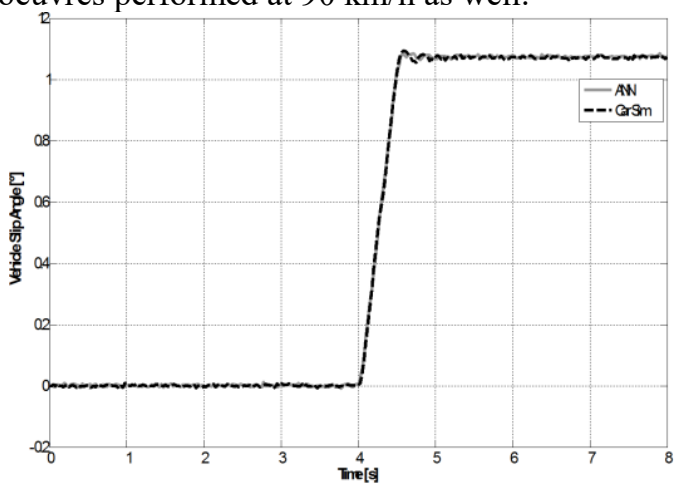

Figure 4. $45^{\circ}$ step steer @ $30 \mathrm{~km} / \mathrm{h}, \mu=1$.

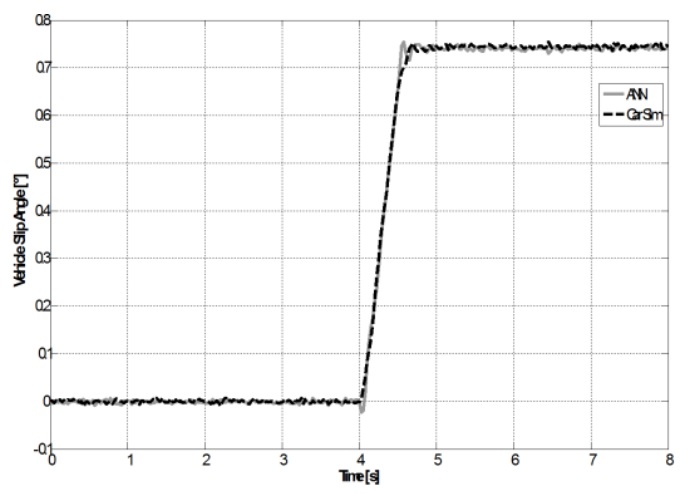

Figure 5. $45^{\circ}$ step steer $@ 60 \mathrm{~km} / \mathrm{h}, \mu=1$. 


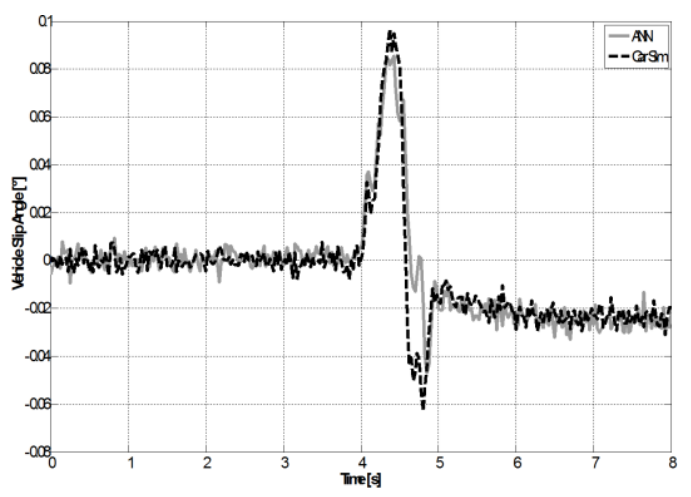

Figure 6. $45^{\circ}$ step steer $@ 90 \mathrm{~km} / \mathrm{h}, \mu=1$.

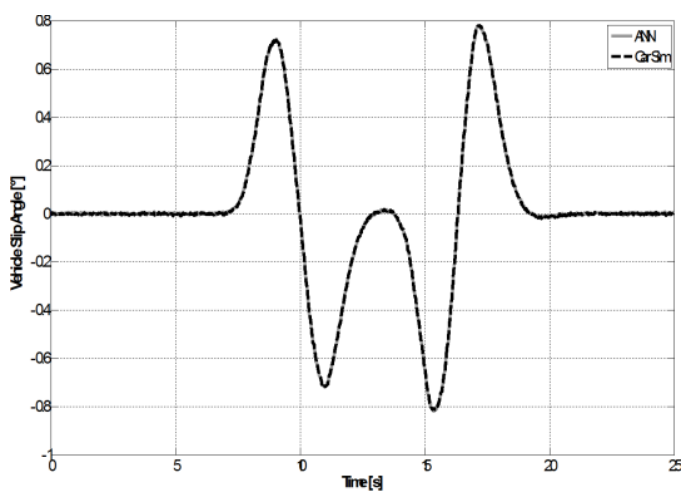

Figure 7. Double lane change @ $30 \mathrm{~km} / \mathrm{h}, \mu=1$.

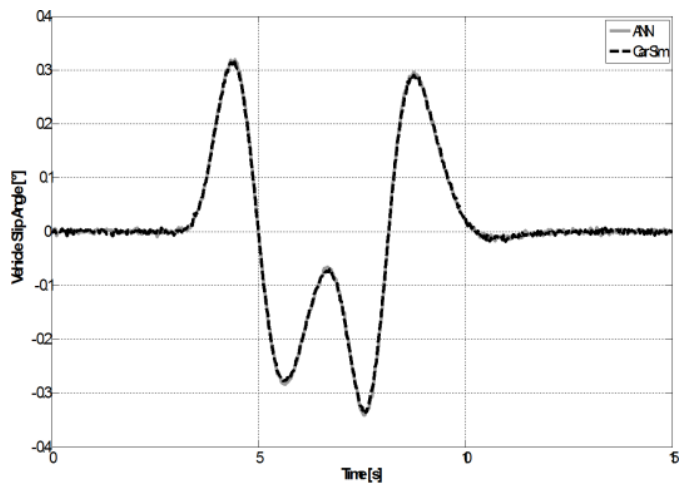

Figure 8. Double lane change @ $60 \mathrm{~km} / \mathrm{h}, \mu=1$.

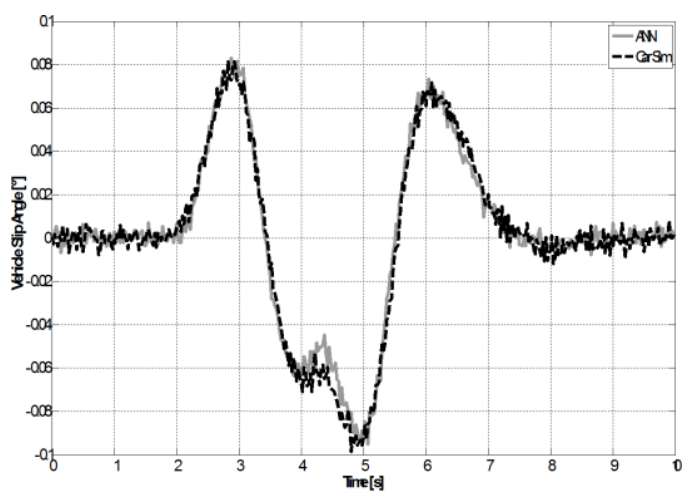

Figure 9. Double lane change @ $90 \mathrm{~km} / \mathrm{h}, \mu=1$.

Test results for $\mu=0.7$ are reported below in figures from 10 to 15 . Test results show that mean and maximum errors are always under 5\%. Once again, best results are in the lower speed range hence with low tyre slip angles.

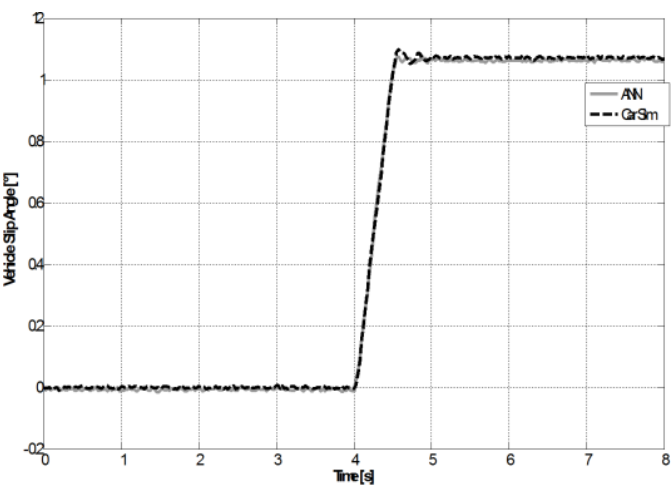

Figure 10. $45^{\circ}$ step steer @ $30 \mathrm{~km} / \mathrm{h}, \mu=0.7$.

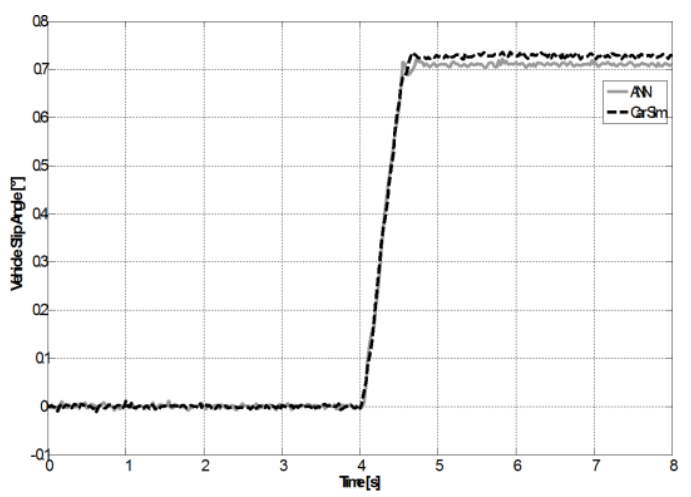

Figure 11. $45^{\circ}$ step steer @ $60 \mathrm{~km} / \mathrm{h}, \mu=0.7$.

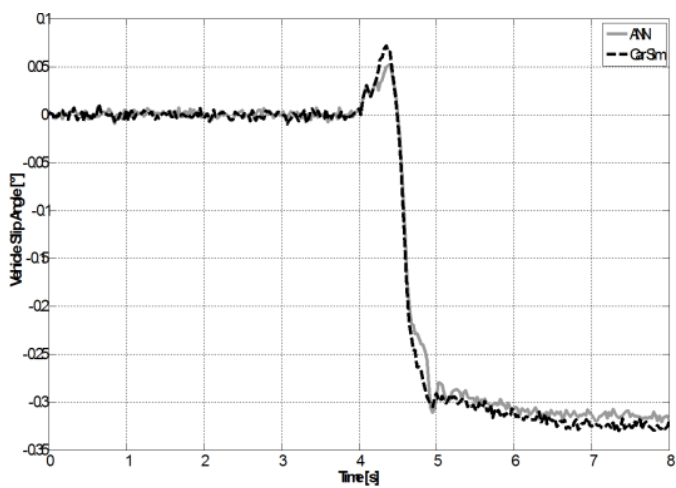

Figure 12. $45^{\circ}$ step steer @ $90 \mathrm{~km} / \mathrm{h}, \mu=0.7$.

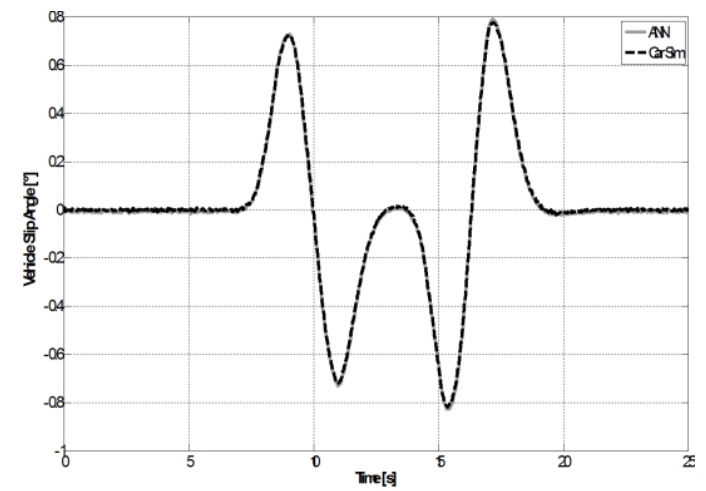

Figure 13. Double lane change @ $30 \mathrm{~km} / \mathrm{h}, \mu=0.7$. 


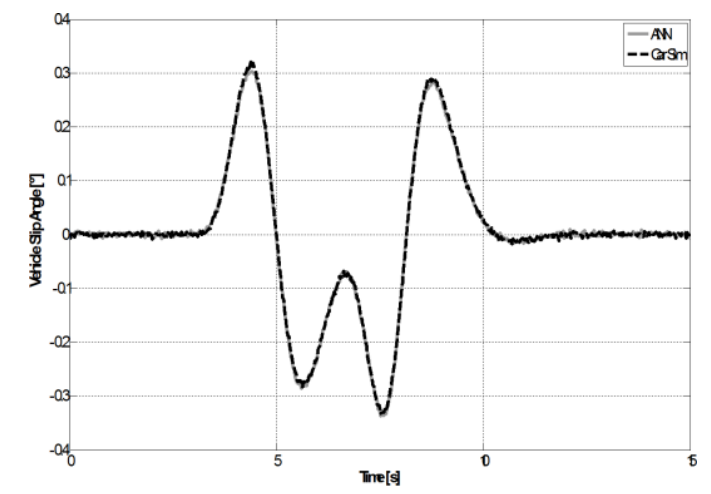

Figure 14. Double lane change @ $60 \mathrm{~km} / \mathrm{h}, \mu=0.7$.

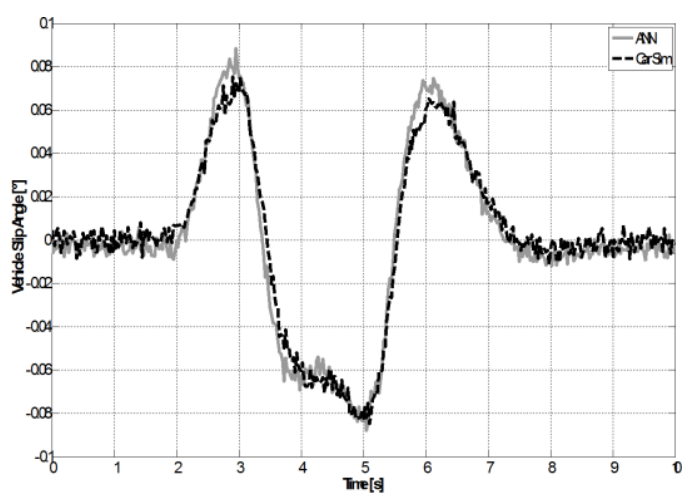

Figure 15. Double lane change @ $90 \mathrm{~km} / \mathrm{h}, \mu=0.7$.

Test results for $\mu=0.4$ are reported below in figures from 16 to 21 . Note that, due to the low adherence conditions, tests have been carried out at $30 \mathrm{~km} / \mathrm{h}, 40$ $\mathrm{km} / \mathrm{h}$ and $50 \mathrm{~km} / \mathrm{h}$. Also in this case, both mean and maximum error between estimated and actual value of the vehicle slip angle are well below 5\% in the lower speed range (see figures 16, 17, 19, 20).

However, tests performed at $50 \mathrm{~km} / \mathrm{h}$ show bigger errors (se figures 18 and 21). It is worth highlighting that a double lane change and step steer performed at $50 \mathrm{~km} / \mathrm{h}$ wit $\mu=0.4$ are very challenging manoeuvres.

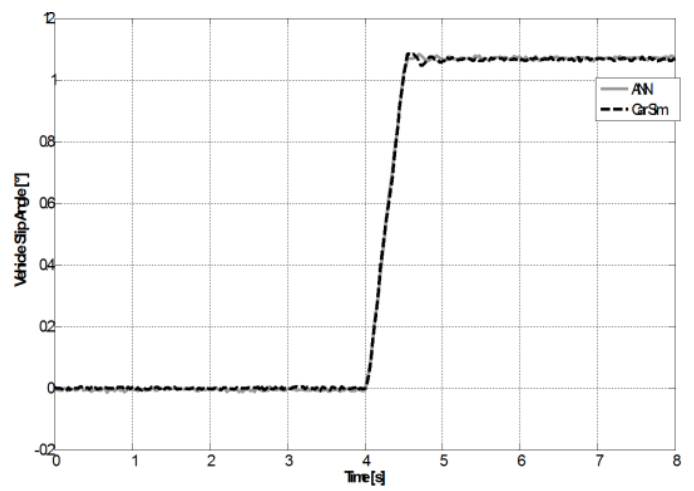

Figure 16. $45^{\circ}$ step steer @ $30 \mathrm{~km} / \mathrm{h}, \mu=0.4$.

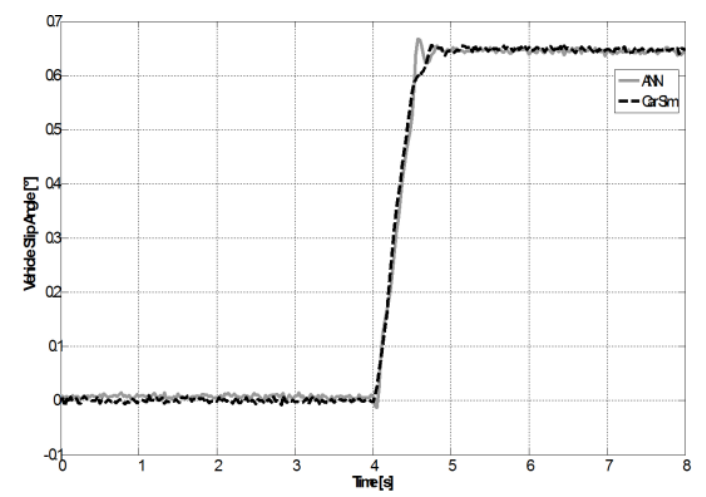

Figure 17. $45^{\circ}$ step steer $@ 40 \mathrm{~km} / \mathrm{h}, \mu=0.4$.

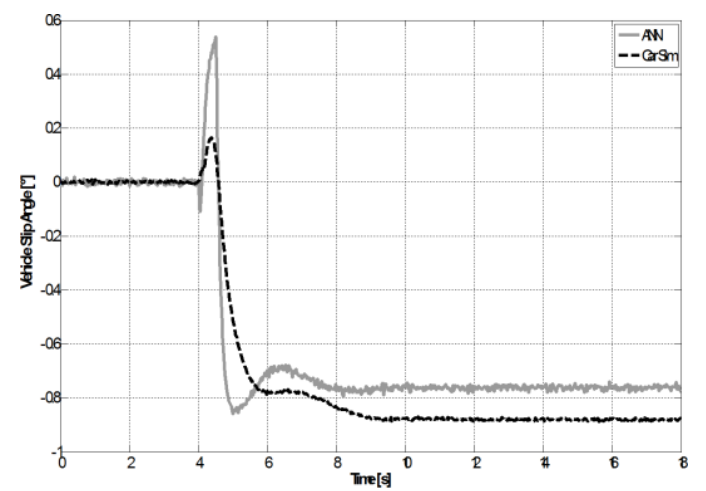

Figure 18. $45^{\circ}$ step steer @ $50 \mathrm{~km} / \mathrm{h}, \mu=0.4$.

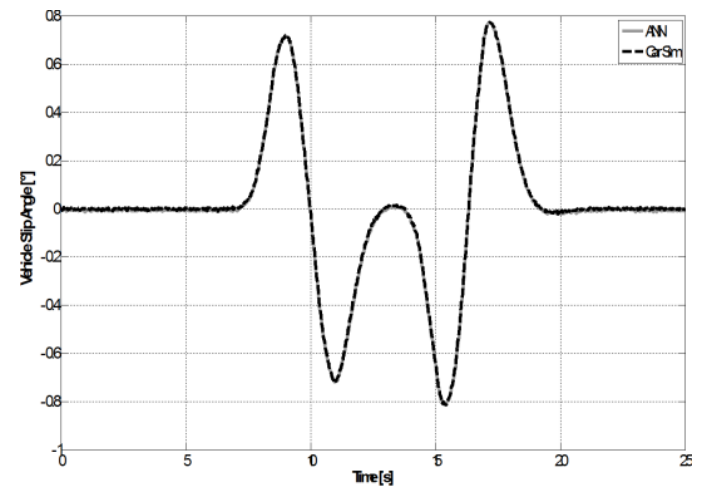

Figure 19. Double lane change @ $30 \mathrm{~km} / \mathrm{h}, \mu=0.4$.

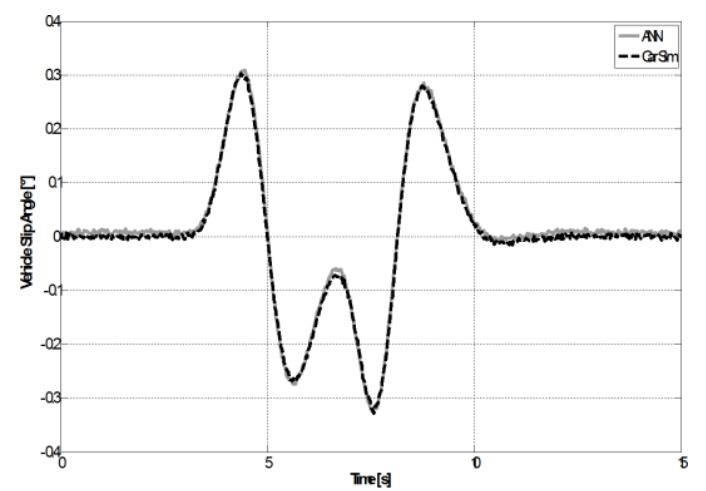

Figure 20. Double lane change @ $40 \mathrm{~km} / \mathrm{h}, \mu=0.4$. 


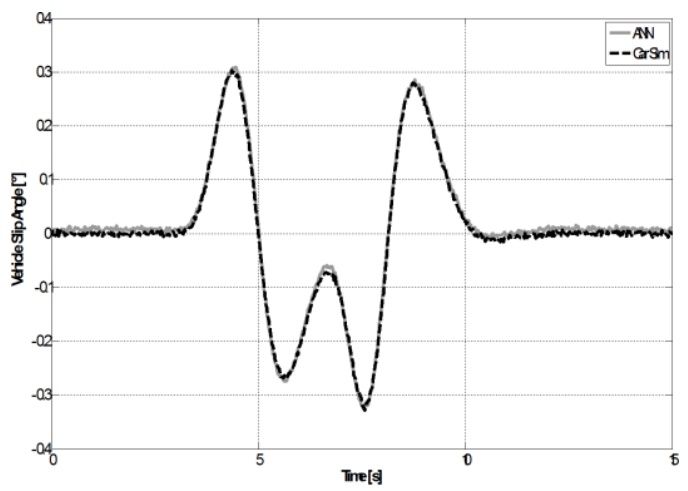

Figure 21. Double lane change @ $50 \mathrm{~km} / \mathrm{h}, \mu=0.4$.

Therefore, results indicate that the proposed ANN is able to accurately learn the above-mentioned training data sets and hence, well trained. Indeed, the trained ANN is able to generalize a wide range of operating conditions and the predictive ability of this network for vehicle side-slip angle is satisfactory.

Finally, for generic pattern identification the network takes $1.34 \mathrm{e}-5 \mathrm{~s}$ (calculated using MATLAB ${ }^{\circledR}$ ), hence the proposed trained ANN is able to perform real-time calculations. Increasing the number of neurons in the hidden layer the calculation time grows exponentially without a significant improvement of the estimation.

Table 1. Nomenclature

\begin{tabular}{cc}
\hline Abbreviation & Meaning \\
\hline ESP & electronic stability program \\
DYC & dynamic yaw control \\
ABS & antilock braking system \\
EKF & extended Kalman filter \\
ANN & artificial neural network \\
BP & back-propagation \\
$v x$ & vehicle speed \\
$\delta$ & steering angle \\
$a y$ & vehicle lateral acceleration \\
$a x$ & vehicle longitudinal acceleration \\
$\psi$ & vehicle yaw rate \\
$\beta$ & vehicle side-slip angle \\
MSE & mean squared error \\
DLC & double lane change \\
SS & step steer \\
$\mu$ & friction coefficient \\
\hline
\end{tabular}

\section{Conclusion}

In this paper, a method to estimate vehicle side-slip angle during transients and steady state conditions has been presented. The proposed technique is based on a simple artificial neural network able to perform real time estimations.

A custom design network training manoeuvre is proposed in order to keep the network as simple as possible. This approach leads to use a simple 5-10-1 layout therefore reducing the computational burden of the estimation algorithm thus making it suitable for real-time, on-line applications.

Despite the simplicity of the network, simulation results have proved the reliability and effectiveness of the proposed methodology. An accurate comparison with the estimated vehicle side-slip angle is obtained by means of the simulations performed with the CarSim ${ }^{\circledR}$ package. For the sake of completeness, results are summarized in Table 2.

This method allows the user to better evaluate the vehicle behaviour and to understand the critical points regarding stability and active safety even when the direct measurement of the side-slip angle is not available.

Table 2. Results summary.

\begin{tabular}{cccc}
\hline Test & Speed [km/h] & $\boldsymbol{\mu}$ & Mean Error [\%] \\
\hline DLC & 30 & 1 & 1.75 \\
DLC & 60 & 1 & 1.92 \\
DLC & 90 & 1 & 4.85 \\
DLC & 30 & 0.7 & 1.55 \\
DLC & 60 & 0.7 & 1.63 \\
DLC & 90 & 0.7 & 4.96 \\
DLC & 30 & 0.4 & 2.12 \\
DLC & 40 & 0.4 & 2.67 \\
DLC & 50 & 0.4 & 3.32 \\
SS & 30 & 1 & 1.98 \\
SS & 60 & 1 & 1.95 \\
SS & 90 & 1 & 3.60 \\
SS & 30 & 0.7 & 1.88 \\
SS & 60 & 0.7 & 2.98 \\
SS & 90 & 0.7 & 3.58 \\
SS & 30 & 0.4 & 1.76 \\
SS & 40 & 0.4 & 2.15 \\
SS & 50 & 0.4 & 15.78 \\
\hline
\end{tabular}

\section{References}

1. Marchesin, F.P.; Barbosa, R.S.; Alves, M.A.L.; Gadola, M.; Chindamo, D.; Benini, C. (2016). Upright mounted pushrod: the effects on racecar handling dynamics. The Dynamics of Vehicles on Roads and Tracks. Proceedings of the 24th Symposium of the International Association for Vehicle System Dynamics, IAVSD 2015. 543-552.

2. Benini, C; Gadola, M; Chindamo, D; Uberti, S; Marchesin, F.P.; Barbosa, R.S. (2017). The influence of suspension components friction on race car vertical dynamics. Vehicle System Dynamics. 55(3): 338-350.

3. Gadola, M., Chindamo, D., Romano, M., Padula, F., Development and Validation of a Kalman FilterBased model for Vehicle Slip Angle Estimation, Vehicle System Dynamics, 2004, 52(1), 68-84.

4. H. Du, J. Lam, K.-C. Cheung W. Li, N. Zhang, Sideslip angle estimation and stability control for a vehicle with a non-linear tyre model and a varying speed, Proceedings of the Institution of Mechanical 
Engineers, Part D: Journal of Automobile Engineering, 229 (2015) 486-505.

5. D.W. Pi, N. Chen, J.X. Wang, B.J. Zhang, Design and evaluation of sideslip angle observer for vehicle stability control, International Journal of Automotive Technology, 12 (2011) 391-399.

6. Chindamo, D., Economou, J.T., Gadola, M., Knowles, K., A neurofuzzy-controlled power management strategy for a series hybrid electric vehicle, Proceedings of the Institution of Mechanical Engineers, Part D: Journal of Automobile Engineering, 2014, 228(9), 1034-1050.

7. A. Lucas, M. Duran, M. Carmona, M. Lapuerta, Modeling diesel particulate emissions with neural networks, Fuel 4 (2001) 548-593.

8. M. Gwak, K. Jo, M. Sunwoo, Neural-network multiple models filter (NMM)-based position estimation system for autonomous vehicles, International Journal of Automotive Technology, 14 (2013) 265-274.

9. V. Cirovic, D. Aleksendric, D. Smiljanic, Longitudinal wheel slip control using dynamic neural networks, Mechatronics, 23 (2013) 135-146.

10. Crema, C.; Depari, A.; Flammini, A.; Vezzoli, A.; Benini, C.; Chindamo, D.; Gadola, M.; Romano, M. (2015). Smartphone-based system for vital parameters and stress conditions monitoring for nonprofessional racecar drivers. Proceedings of the 2015 IEEE SENSORS. 7370521.

11. M. Gadola, D. Vetturi, A. Magalini, Use of an innovative multi-input one-output neural network for experimental data numerical description, 7th International Research/Expert Conference - TMT 2003, Lloret de Mar, Barcelona, Spain, September 2003.

12. H.Sasaki, T.Nishimaki, A side-slip angle estimation using neural network for a wheeled vehicle, SAE Technical Paper 2000-01-0695, 2000.

13. Wang Wei, Bei Shaoyi, Zhang Lanchun, Zhu Kai, Wang Yongzhi, and HangWeixing, Vehicle Sideslip Angle Estimation Based on General Regression Neural Network, Mathematical Problems in Engineering, Volume 2016, Article ID 3107910, 7 pages.

14. Dal Bianco, N.; Lot, R.; Gadola, M., Minimum time optimal control simulation of a GP2 race car. Proceedings of the Institution of Mechanical Engineers, Part D: Journal of Automobile Engineering. Article in press.

15. M. Kato, K. Isoda, H. Yuasa, Estimation of vehicle slip angle with artificial neural network. SAE Technical paper, 15 (1994) 73-86.

16. S. Melzi, E. Sabbioni, On the vehicle sideslip angle estimation through neural networks. Mechanical Systems and Signal Processing, 25 (2011) 2005-2019.

17. P.D. Wesserman, Neural Computing Theory and Practice, Van Nostrand Reinhold, 1989.

18. W-J. QI, P. Zhang, Z-L. Deng, Robust Sequential Covariance Intersection Fusion Kalman Filtering over Multi-agent Sensor Networks with Measurement Delays and Uncertain Noise Variances, Acta Automatica Sinica, 40 (2014) 2632-2642.
19. A. Parlak, Y. Islamoglu, H. Yasar, A. Egrisogut, Application of Artificial Neural Network to Predict Specific Fuel Consumption and Exhaust Temperature for a Diesel Engine, Applied Thermal Engineering, 26 (2006) 824-828.

20. M.T. Hagan, H.B. Demuth, M. Beale, Neural Network Design, PWS Publishing Company, Boston, 1995.

21. Levenberg, K., A Method for the Solution of Certain Problems in Least-Squares, Quarterly Applied Math. 2, pp. 164-168, 1944.

22. Marquardt, D., "An Algorithm for Least-Squares Estimation of Nonlinear Parameters," SIAM Journal Applied Math., Vol. 11, pp. 431-441, 1963.

23. M. Aydinalp, V.I. Ugursal, A.S. Fung, Predicting residential appliance, lighting, and space cooling energy consumption using neural networks. The Fourth International Thermal Energy Congress, Cesme, Turkey, July.

24. S. Haykin, Neural Networks, A comprehensive foundation, McMillian College Publishing Company, New York, 1994. 